\title{
Comparison of Vapor Phase and Liquid Phase Oxidation of Ethylene to Acetaldehyde with Palladium Chloride-Active Charcoal Catalyst*
}

\author{
by Kaoru Fujimoto** and Taiseki Kunugi**
}

\begin{abstract}
Summary: Comparative studies on the vapor phase and liquid phase oxidation of ethylene were made with palladium chloride-active charcoal catalyst. The catalyst activity in the vapor phase reaction was higher than that in the liquid phase reaction. Acetaldehyde was obtained with high selectivity in both phases. The rate of acetaldehyde formation in the vapor phase was accelerated by the increase in steam pressure and was suppressed by the rise in the reaction temperature while the rate in the liquid phase reaction was accelerated by the rise in the reaction temperature. Aqueous hydrogen chloride solution of about $0.1 \mathrm{~N}$ was effective as a reaction medium to keep the catalyst activity constant. Contrary to the vapor phase reaction the rate in the liquid phase reaction decreased with the increase in partial pressure of oxygen.
\end{abstract}

\section{Introduction}

Oxidation of olefins with an aqueous acidic solution of palladium chloride has been known since 1895. J. Smidt et al. have discovered a new catalyst composed of palladium chloride and cupric chloridel) for oxidizing olefins by oxygen to the corresponding saturated carbonyl compounds.

The authors have discovered that when palladium salts adsorbed on active charcoal are employed as catalysts olefins are catalytically oxidized by oxygen to form the corresponding carbonyl compounds without requiring any redox reagents such as cupric chloride ${ }^{2)}$. Catalysis of this reaction consists of (a) the oxidation of olefins by a palladium salt in the presence of steam and (b) the re-oxidation of reduced palladium by oxygen catalyzed by active charcoal ${ }^{3}$. Authors have clarified several characteristic features and mechanisms of the vapor phase reaction ${ }^{1), 8)}$. This catalyst was found to be also effective for the catalytic synthesis of vinyl acetate from ethylene, oxygen and acetic acid4).

In this paper, a comparative study of the oxidation of ethylene in the vapor and liquid phases was made in order to understand this catalytic reaction more in detail.

* Received November 20, 1972.

** Department of Synthetic Chemistry, Faculty of Engineering, University of Tokyo (Hongo, Bunkyo$\mathrm{ku}$, Tokyo 113)

\section{Experimental}

Vapor phase reactions were conducted in a conventional flow type reactor under atmospheric pressure. The reactor was a glass tube (20 mm I.D. $\times 450 \mathrm{~mm}$ L.) equipped with a thermo-well along the central axis and with a heating jacket. The catalyst charged was from 1 to $10 \mathrm{~g}$.

Liquid phase reactions were carried out in a cylindrical bubble type reactor $(25 \mathrm{~mm}$ I.D. $\times$ $450 \mathrm{~mm} \mathrm{~L}$.) with a gas distribution plate made of sintered glass at the bottom, and the powdered catalyst was agitated by bubbling feed gas to keep a uniform dispersion under atmospheric pressure.

Acetaldehyde was determined by titration with hydroxylamine hydrochloride after its absorption in water. Other products were analyzed by gas-chromatography (silica gel column for $\mathrm{O}_{2}, \mathrm{CO}, \mathrm{CO}_{2}$ and ethylene; di-nonyl phthalate on celite column for butenes and methyl ethyl ketone).

Catalyst preparation: Palladium chloride was dissolved in dilute hydrochloric acid and active charcoal was added, which was previously boiled with $10 \%$ nitric acid and washed with water. After $1 \sim 2$ days, palladium chloride was adsorbed completely on the active charcoal. This palladium salt supported charcoal was washed with a small amount of water, followed by drying in vacuum at approximately $150^{\circ} \mathrm{C}$. for 5 hours.

Volume 15, No. 1, May 1973 
The active charcoals used were commercially available and they were made from wood by steam activation. BET surface area of the charcoal for vapor phase oxidation was 1,510 $\mathrm{m}^{2} / \mathrm{g}$, and that for liquid phase oxidation was $1,310 \mathrm{~m}^{2} / \mathrm{g}$. Both charcoals showed nearly the same catalytic activity in the vapor phase reaction when they carried the same amount of palladium chloride. The content of palladium chloride and catalyst size were $1.6 \mathrm{wt} \%$ and $i \sim 2 \mathrm{~mm}$ for vapor phase reaction, and $0.8 \mathrm{wt} \%$ and $3.9 \mu$ for liquid phase reaction, respectively.

\section{Results and Discussion}

The main product of the reaction was acetaldehyde, and some minor amounts of carbon dioxide and butenes were formed. In addition to these by-products, a trace of methyl ethyl ketone (in the vapor phase oxidation) and ethyl chloride (in the liquid phase oxidation) were detected.

\subsection{Activity Change with Time}

In the liquid phase reaction, as shown in Fig. 1, a marked decrease in activity was observed when water was used as a reaction medium, while catalytic activity did not decrease when $0.1 \mathrm{~N}$ hydrochloric acid was used instead of water. The amount of acetaldehyde formed in 12 hours was about 120 moles per mole of palladium chloride supported on active charcoal.

This result is explained as the interaction between the reduced palladium $\left(\mathrm{Pd}^{\circ}\right)$ and the chloride anion formed by the Reaction (1),

$$
\mathrm{PdCl}_{2} \rightarrow \mathrm{Pd}^{\circ}+2 \mathrm{Cl}^{-}
$$

is very weak and, therefore, the chloride anion

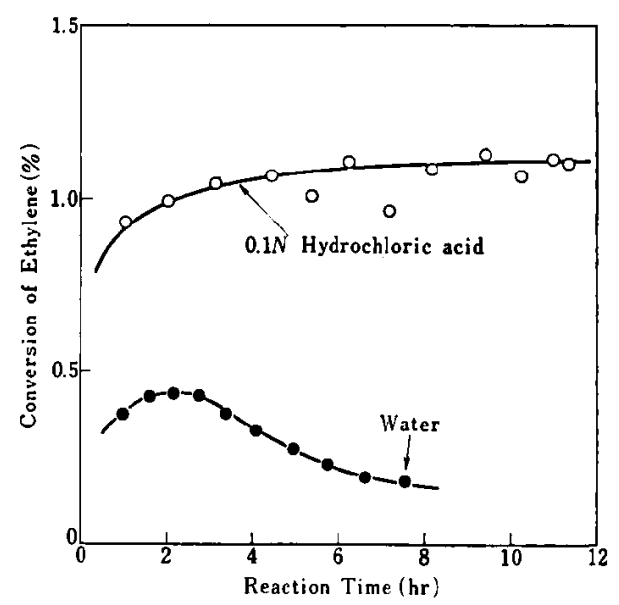

Liquid $150 \mathrm{ml}$, Catalyst $5.0 \mathrm{~g}, \mathrm{C}_{2} \mathrm{H}_{4}: \mathrm{O}_{2}$ $2: 1$, Feed rate $105 \mathrm{ml} / \mathrm{min}, 70^{\circ} \mathrm{C}$

Fig. I Effect of Hydrogen Chloride on Liquid Phase Reaction Rate can easily diffuse into the water. The reduced palladium which has lost chloride anion can no longer exist stably and aggregates to form palladium metal. The oxidation rate of this palladium metal to palladium chloride is very slow, and thus the catalytic activity decreases irreversibly. In fact, a significant amount of chloride anion was detected in the water medium after several hours of reaction.

In the vapor phase reaction, the activity was confirmed to be substantially constant over 100 hours of operation.

\subsection{Effect of Temperature}

Fig. 2 shows the effect of reaction temperature in the vapor and liquid phase reactions. It should be noted that the reaction rate in the liquid phase increased with the rise in temperature, giving the apparent activation energy of 8.6 $\mathrm{kcal} / \mathrm{mol}$, and the selectivity of acetaldehyde also increased from about $80 \mathrm{~mol} \%$ at $50^{\circ} \mathrm{C}$ to about $96 \mathrm{~mol} \%$ at $90^{\circ} \mathrm{C}$. In the vapor phase, however, the rate decreased significantly with the rise in temperature giving the apparent activation energy of $-18 \mathrm{kcal} / \mathrm{mol}$, and the selectivity of acetaldehyde also decreased, for example, from $97 \mathrm{~mol} \%$ at $65^{\circ} \mathrm{C}$ to $75 \mathrm{~mol} \%$ at $105^{\circ} \mathrm{C}$. The by-products formed were carbon dioxide in the liquid phase reaction and butenes in the vapor phase reaction.

These results may be explained as follows: The elementary reactions of ethylene oxidation by a palladium salt are represented by the following Equations (2) to (5), as postulated by Dozono et al.5) or by Henry6).

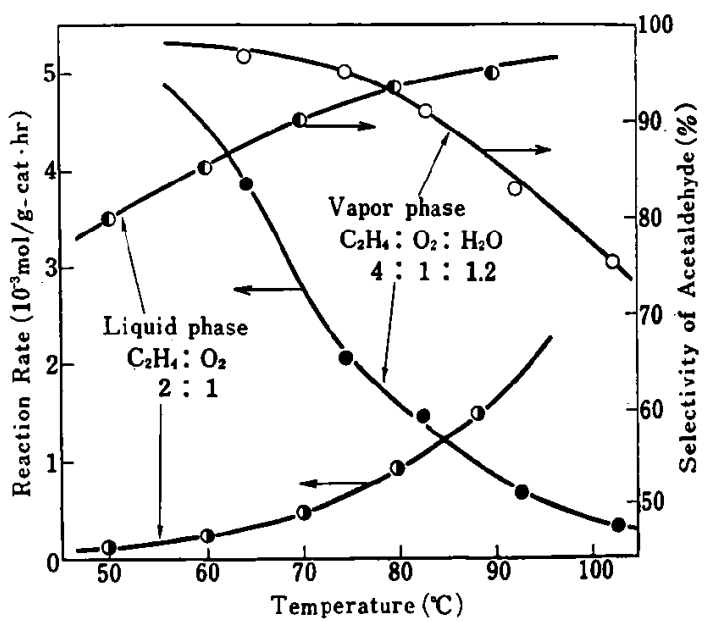

Feed rate : Vapor phase : W/F 11 g-cat $\cdot \mathrm{hr} / \mathrm{mol}$ Liquid phase : $105 \mathrm{ml} / \mathrm{min}(0.1 \mathrm{~N} \mathrm{HCl}$ $150 \mathrm{ml}$, Catalyst $5.0 \mathrm{~g}$ )

Fig. 2 Effect of Reaction Temperature

Bulletin of The Japan Petroleum Institute 


$$
\begin{aligned}
& \mathrm{PdX}_{2}+\mathrm{H}_{2} \mathrm{O} \stackrel{K_{1}}{\rightleftarrows} \mathrm{PdX}_{2}\left(\mathrm{H}_{2} \mathrm{O}\right) \\
& \mathrm{PdX}_{2}\left(\mathrm{H}_{2} \mathrm{O}\right)+\mathrm{C}_{2} \mathrm{H}_{4} \stackrel{K_{2}}{\rightleftarrows} \mathrm{PdX}_{2}\left(\mathrm{H}_{2} \mathrm{O}\right)\left(\mathrm{C}_{2} \mathrm{H}_{4}\right. \\
& \quad \mathrm{PdX}_{2}\left(\mathrm{H}_{2} \mathrm{O}\right)\left(\mathrm{C}_{2} \mathrm{H}_{4}\right)+\stackrel{K_{3}}{\longrightarrow} \mathrm{PdX}_{2}(\mathrm{OH})\left(\mathrm{C}_{2} \mathrm{H}_{4}\right)^{-} \\
& \quad+\mathrm{H}_{3} \mathrm{O}^{+} \\
& \mathrm{PdX}_{2}(\mathrm{OH})\left(\mathrm{C}_{2} \mathrm{H}_{4}\right)^{-} \underset{k}{\stackrel{\mathrm{H}_{2} \mathrm{O}}{\rightarrow} \mathrm{CH}_{3} \mathrm{CHO}+\mathrm{Pd}^{\circ}} \\
& \quad+2 \mathrm{HX}+\mathrm{OH}^{-}
\end{aligned}
$$

Thus, at least two molecules of water are necessary for the oxidation reaction. Fig. 3 shows the conspicuous effect of partial pressure of steam in the vapor phase reaction. As shown in Equations $(2) \sim(4)$, the co-ordination of water molecules to palladium is necessary as ligands prior to reacting with ethylene. Since the coordination reaction is generally exothermic, the equilibrium in each of Equations (2) (4) moves to the left side with the rise in temperature; therefore, the amount of the co-ordinated water molecules decreases with rising temperature.

When the reaction is conducted in the liquid phase, there is a sufficient amount of water molecules around the palladium salts and, therefore, the amount of co-ordinated water molecules is substantially independent of the reaction temperature. Thus the temperature dependency of the liquid phase reaction is analogous to that of the conventional Wacker reaction?).

\subsection{Effects of Concentration and Partial \\ Pressure of Reactants}

In the papers already cited ${ }^{5), 6)}$, several water molecules have been considered to take part directly in the formation of acetaldehyde. However, the influence of water pressure on reaction rate has not been clarified in the homogeneous

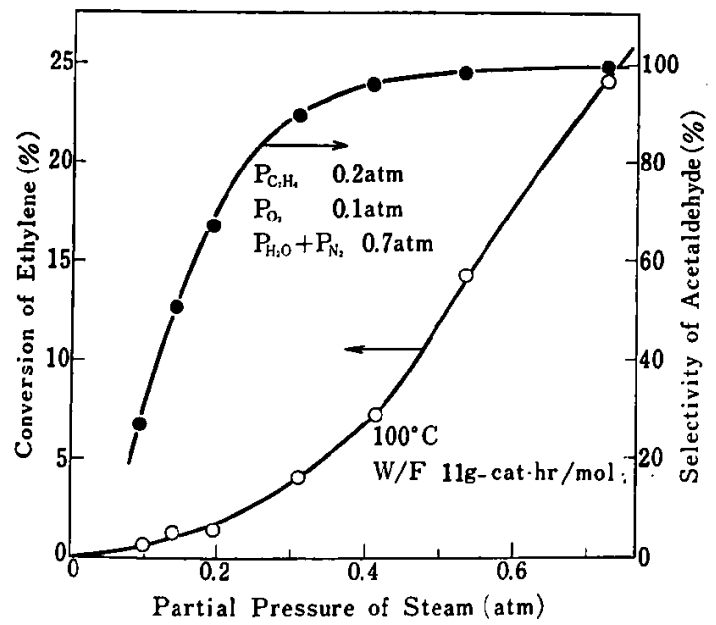

Fig. 3 Effect of Partial Pressure of Steam in Vapor Phase Reaction system because of the presence of excess water.

The authors have already shown that steam pressure has a marked effect on the acceleration of the acetaldehyde formation as shown in Fig. 3 in the vapor phase reaction, and the order of reaction rate with respect to steam pressure is experimentally obtained as about $3^{8}$ ). Thus the rate expression (6) is derived for ethylene oxidation on the basis of the reaction mechanisms $(2) \sim(5)$.

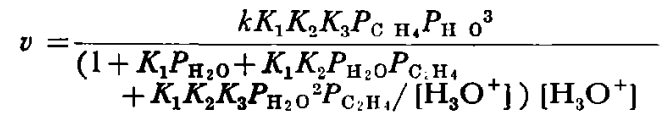

Comparing Equation(6) with the experimental results, it is concluded that the co-ordination of water molecules to palladium is not so strong and consequently $K_{1}$ is small. Equation (6), then, can be rewritten by the approximate rate expression (7).

$$
v=k K_{1} K_{2} K_{3} P_{\mathrm{C}_{2} \mathrm{H}_{4}} P_{\mathrm{H}_{2} \mathrm{O}^{3}} /\left[\mathrm{H}_{3} \mathrm{O}^{+}\right]
$$

Equation (7) suggests that the apparent activation energy is smaller than zero if the sum of the heat liberated by co-ordination of water molecules and ethylene according to Equations (2) to (4) is larger than the activation energy of Equation (5).

The effect of the concentration of hydrogen chloride on reaction rate in the liquid phase is given in Fig. 4, which shows a severe suppression at higher concentrations. The order of reaction rate with respect to the concentration of hydrogen chloride is between -1 and -2 , while

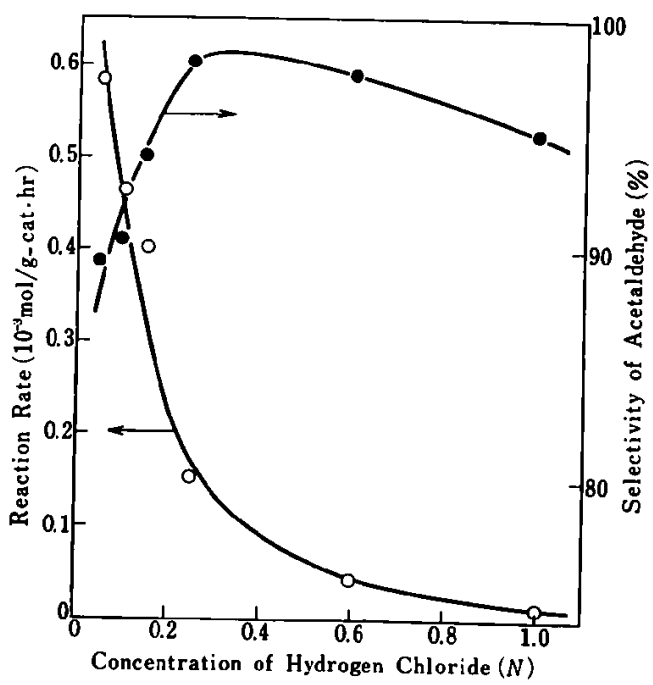

Liquid $150 \mathrm{ml}$, Catalyst $5.0 \mathrm{~g}, \mathrm{C}_{2} \mathrm{H}_{\mathbf{4}}: \mathrm{O}_{2} 2: 1$, Feed rate $105 \mathrm{ml} / \mathrm{min}, 70^{\circ} \mathrm{C}$

Fig. 4 Suppression of Reaction Rate by Hydrogen Chloride in Liquid Phase Reaction

Volume 15, No. 1, May 1973 


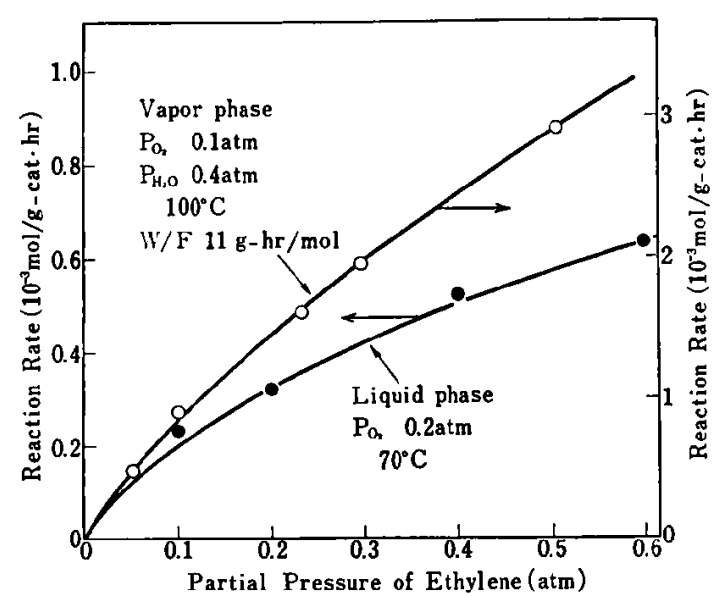

Liquid phase : $0.1 \mathrm{~N} \mathrm{HCl} 150 \mathrm{ml}$, Gatalyst $5.0 \mathrm{~g}$, Feed rate $105 \mathrm{ml} / \mathrm{min}$

Fig. 5 Effect of Partial Pressure of Ethylene

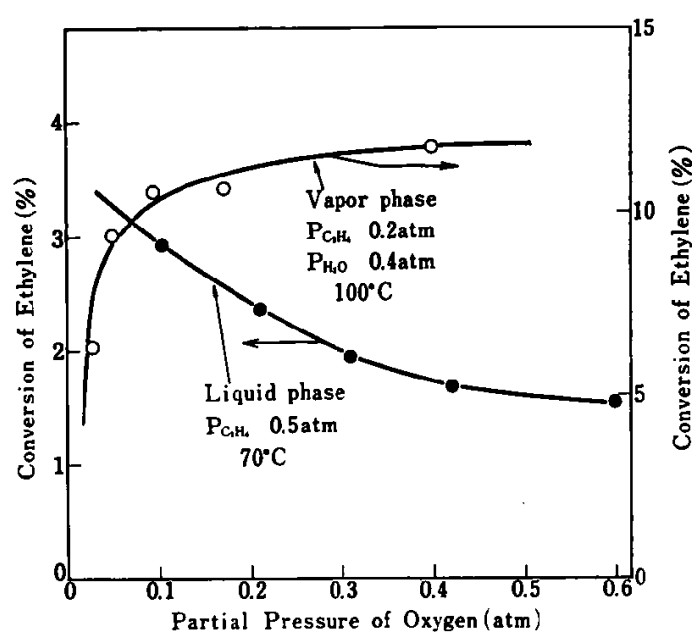

Vapor phase : Catalyst $5.0 \mathrm{~g}$, Feed rate $168 \mathrm{ml} / \mathrm{min}$ Liquid phase : $0.1 \mathrm{~N} \mathrm{HCl} 150 \mathrm{ml}$, Catalyst $5.0 \mathrm{~g}$, Feed rate $105 \mathrm{ml} / \mathrm{min}$

Fig. 6 Effect of Partial Pressure of Oxygen

that of Wacker reaction is -3.0 . The reason for such a difference is not yet clear.

The effect of partial pressure of ethylene on the reaction rate is shown in Fig. 5. The rate equation derived from it is

$$
v=A P_{\mathrm{C}_{2} \mathrm{H}_{4}} /\left(B+C P_{\mathrm{C}_{2} \mathrm{H}_{4}}\right)
$$

where $A, B$ and $C$ are constant.

As shown in Fig. 6, the effect of partial pressure of oxygen on the rate of liquid phase reaction is remarkably different from that of vapor phase reaction. The reaction rate increases, in the vapor phase, with the increase in oxygen pressure, while the rate of liquid phase reaction decreases with the increase in oxygen pressure. The effect of oxygen in the vapor phase reaction is understood as oxygen promoting the reaction

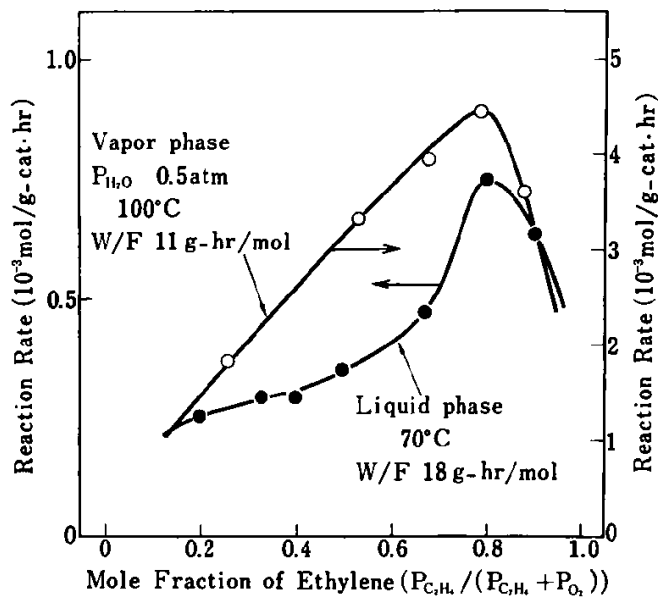

Fig. 7 Effect of Molar Ratio of Ethylene to Oxygen

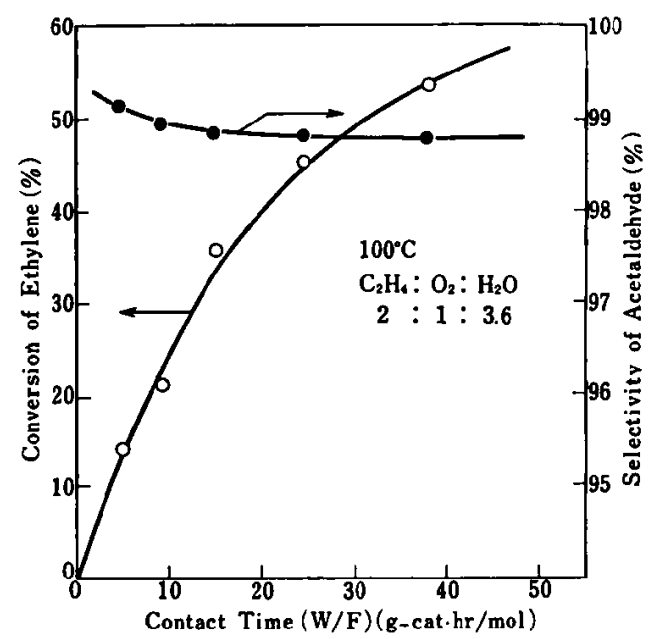

Fig. 8 Effect of Contact Time on Vapor Phase Reaction

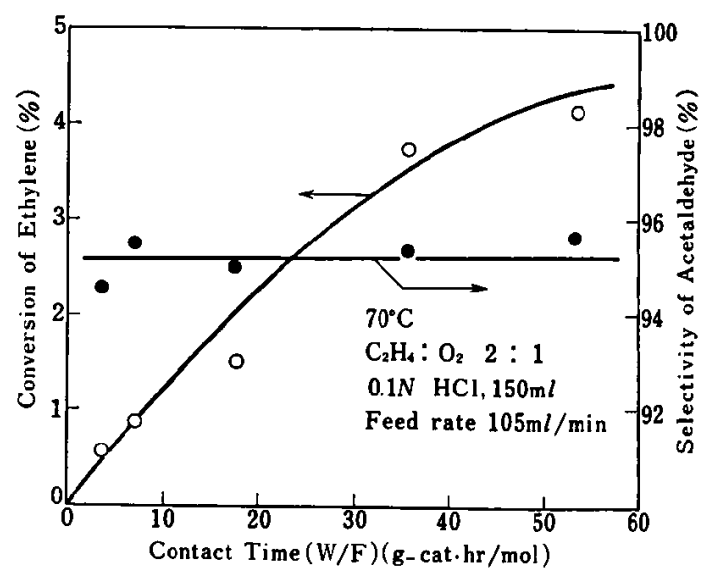

Fig. 9 Effect of Contact Time on Liquid Phase Reaction

$\mathrm{Pd}^{\circ} \rightarrow \mathrm{Pd}^{2+}$; consequently the overall reaction rate increases with increasing oxygen pressure. In the liquid phase reaction, however, the rate is represented as $v=\frac{A^{\prime}}{1+B^{\prime} P_{0_{2}}}+C^{\prime}$, where $A^{\prime}$, Bulletin of The Japan Petroleum Institute 
$B^{\prime}$ and $C^{\prime}$ are constant. This suggests that oxygen co-ordinates with the active sites on the catalyst to prevent the co-ordination of ethylene or water. The reason for such effect of oxygen in the liquid phase is not yet understood.

Fig. 7 shows the rate of formation of acetaldehyde against the mole ratio of ethylene to oxygen. In both phases the maximum rate is obtained at the mole ratio of ethylene to oxygen $4: 1$.

Effects of contact time are illustrated in Fig. 8 and Fig. 9. Such a slow reaction rate in the liquid phase seems to be caused by lower reaction temperature, lower palladium ion concentration and suppression by oxygen as mentioned above. Selectivity of acetaldehyde is about 98 to $99 \mathrm{~mol} \%$ in the vapor phase reaction and about 95 to $96 \mathrm{~mol} \%$ in the liquid phase reaction, and is affected little by contact time of the feed gas mixture in both cases. The high selectivity of acetaldehyde even in contact for a long time is due to the slow oxidative decomposition of acetaldehyde to carbon dioxide.

\section{References}

1) Smidt, J., Hafner, W., Jira, R., Sedlmeir, J., Sieber, R., Rüttinger, R., Kojer, H., Angew. Chem., 71, 176 (1959).

2) Fujimoto, K., Negami, Y., Kunugi, T., Kyogo Kagaku Zasshi, 73, 1822 (1970).

3) Fujimoto, K., Takahashi, T., Negami, Y., Kunugi, T., ibid., 74, 1076 (1971).

4) Kunugi, T., Arai H., Fujimoto, K., Bull. Japan Petrol. Inst., 12, 97 (1970).

5) Dozono, T., Shiba T., ibid., 5, 8 (1963).

6) Henry, P. M., J. Am. Chem. Soc., 86, 3246 (1964).

7) Teramoto, K., Oga, T., Kikuchi, S., Ito, M., J. Synthetic Org. Chem., 21, 298 (1963).

8) Fujimoto, K., Takeda, H., Negami, Y., Kunugi, T., Kogyo Kagaku Zasshi, 74, 375 (1971).

9) Jira, R., Sedlmeier, J., Smidt, J., Ann., 693, 99 (1966). 